\title{
Conceptual Study on Non-ablative TPS for Hypersonic Vehicles
}

\author{
Zonglin Jiang ${ }^{1}$, Yunfeng Liu and Guilai Han \\ Laboratory of High-temperature Gas Dynamics, Institute of Mechanics, CAS, Beijing, 100190, China
}

\begin{abstract}
In order to achieve efficient wave drag reduction under non-zero attack angles and avoid the severe aerodynamic heating, a new concept of the Non-ablative Thermal Protection System (NaTPS) for hypersonic vehicles was proposed based on the idea that the conical shock wave angle can be enlarged by lateral jets to push the conical shock away from the blunt body surface. In the NaTPS, a spike-blunt body structure and lateral jets are combined together to develop a new shock-reconstructing system in front of hypersonic vehicles. When the spike acts to recast the bow shock in front of a blunt body into a conical shock, the lateral jet works to protect the spike tip from overheating and push the conical shock away from the blunt body when a pitching angle exists during flight. The experimental flow visualization and the pressure measurements were conducted in a hypersonic wind tunnel for both the conceptual demonstration and CFD validation. Numerical simulations were also carried out to examine the complex flow around the NaTPS. Both experimental and numerical results show that the NaTPS works well for shock drag reduction and thermal protection. The shock/shock interaction on shoulders of the blunt body is avoided due to lateral jet injection and the peak pressure at the reattachment region is reduced by $65 \%$ under a $4 \circ$ attack angle. The lateral jet could be powered either by high pressure gas stored in the tank or by the water evaporation process in which water absorbs the heat from the hot walls of the blunt noses. The jet pressure needed for producing lateral jet is much smaller than for the forward-facing jet from the stagnation point. The advantages of this concept are well demonstrated and its practical application appears promising.
\end{abstract}

\section{Nomenclature}

$=$ outer diameter of the blunt body $(\mathrm{mm})$

$=$ spike length $(\mathrm{mm})$

$=$ outer diameter of spike $(\mathrm{mm})$

$=$ the coming flow Mach number

$=$ total injection pressure

\section{Introduction}

$\mathrm{T}$

he shock-induced aerodynamic drag and the severe heat transfer are two major issues for the successful development of hypersonic vehicles ${ }^{1}$. The shock wave drag may occupy about two thirds of the total drag of cruising hypersonic vehicles, and the $1 \%$ overall drag reduction will translate to the $5-10 \%$ increase in payload ${ }^{2}$. Moreover, the reduction of shock-induced drag results in temperature decrease in the aerothermal environment around hypersonic vehicles and the Thermal Protect System (TPS) will benefits from the reduction of heat transfer. Therefore, the shock wave drag reduction for hypersonic vehicles is of significant importance.

A useful mean for shock-induced drag reduction of hypersonic vehicles is to minimize the diameter of the vehicle nose and the leading edges because the conical shock induces the less drag than normal shock waves. However, minimizing the diameter of the leading edge and the vehicle nose could increase dramatically the heat transfer flux on the vehicle surface, which will impose more severe problems for TPS designs. Therefore, exploring new concepts that are able to reduce both the aerodynamic drag and the surface heating flux has been an interesting topic for decades.

\footnotetext{
${ }^{1}$ Professor, E-mail: zljiang@imech.ac.cn.
} 
There are several methods, so far reported, for artificially recasting shock wave formation processes. The most effective one of them is to install a physical spike on the nose of blunt bodies ${ }^{3-15}$. The spike recasts a bow shock into a conical shock that induces the lower pressure region ahead of blunt bodies. Doing so, approximate $50 \%$ drag reduction was predicted under the condition of zero attack angle. However, the spike-blunt body structure becomes ineffective for shock drag reduction if the attack angle is not zero because the separation region in the windward side becomes much smaller and the conical shock interacts with the reattachment shock on the shoulder of blunt bodies. This shock/shock interaction process results in the extremely high pressure at the interaction point, which is much higher than the stagnation pressure ${ }^{4}$. Moreover, the much more severe aerodynamic heating at both the spike tip and the shock/shock interaction point on blunt bodies is induced, and the phenomenon blocks the way for application of the spike concept to hypersonic vehicles.

The forward-facing jet injection into the nose region pushes the bow shock wave away from the blunt body nose without the need of a physical nose spike ${ }^{8-10}$. The gas, liquid, or solid powder can be used for injection and an order of $50 \%$ drag reduction as well as large reduction in aerodynamic heat transfer over most parts of the nose surface except in the region around the jet reattachment are obtained at zero attack angle. In the shock wave reattachment region, the shock/shock interaction invariably increases local pressure and heat flux as same as that of aero-spike. Moreover, there are two important problems encountered in the application of the forward-facing jet to hypersonic vehicles. The first problem is the injection needs a high pressure jet to push the bow shock wave away from the blunt nose. The total pressure ratio of the jet to the free stream flow should be much greater than unity. The second one is that the drag reduction performance strongly depends on the attack angle ${ }^{11}$. Even a $2^{0}$ attack angle will cause large reduction of its performance. These two problems tend to limit its application to the vehicles with extreme directional stability and very small attack angle variations over the flight range ${ }^{11}$.

Other approaches to the wave drag reduction are to use the focused energy deposition. A wide variety of energy deposition techniques were investigated by laser pulse, plasma arcs, microwaves, electron beams, pulsed detonations or explosions, etc ${ }^{16-21}$. When the focused energy is deposited in the upstream region of a blunt body, the extremely hot gas is created instantaneously to push the surrounding gas outward ${ }^{17}$. The expansion leaves behind a core of low density and pressure hot gases. When the low pressure and density gases flow over hypersonic vehicles, shock drag reduction is achieved. If the gas temperature inside the core is sufficiently high to make the vehicle's speed be subsonic, then the bow shock wave is locally eliminated and the wave drag is reduced significantly. It is reported that as much as $96 \%$ drag reduction is obtained ${ }^{17}$. The energy addition can also be produced by localized combustion $^{21}$ and the recent research indicates the concept is more attractive ${ }^{22,23}$. However, the power budget and the system complexity are highly prohibitive for using the energy deposition concept. In addition, the high temperature gas produced by local energy deposition probably imposes a heavier burden on the design of thermal protection systems for hypersonic vehicles.

In order to achieve efficient wave drag reduction under non-zero attack angles and avoid the severe aerodynamic heating problem, a new concept of the Non-ablative Thermal Protection System (NaTPS) was proposed based on the idea that the conical shock angle can be enlarged by lateral jets to push the conical shock away from the blunt body surface and make the shock/shock interaction point not occur at the blunt body ${ }^{24}$. Doing so, a spike-blunt body structure and lateral jets are combined together to develop a new shock-reconstructing system for hypersonic vehicles. When the spike acts to recast the bow shock in front of a blunt body into a conical shock, the lateral jet works to protect the spike tip from overheating and push the conical shock away from the blunt body when a pitching angle exists during flight. The experimental flow visualization and the pressure measurements were conducted in a hypersonic wind tunnel for both the conceptual demonstration and the CFD validation. Numerical simulations were carried out to examine the complex flows around the NaTPS. Both numerical results and experimental results show that the NaTPS works well for both shock drag reduction and thermal protection. The shock/shock interaction on shoulders of the blunt body is avoided due to lateral injection and the peak pressure at the reattachment point is reduced by $65 \%$ under a $4 \circ$ attack angle. The lateral jet could be powered either by high pressure gas stored in the tank or by the water evaporation process in which water absorbs the heat from the hot walls of the blunt body noses. The jet pressure needed for producing lateral jet is much smaller than for the forwardfacing jet from the stagnation point. The advantage of this concept is well demonstrated and some results are presented in this paper.

\section{Experiment and numerical descriptions}

The new concept of NaTPS proposed for hypersonic vehicles is schematically shown in Fig.1 (a) and its test model is presented in Fig.1 (b). In the NaTPS, the coolant is stored inside the blunt body to absorb the aerodynamic heat from the coming flows. The gas generated from coolant evaporation flows forward along the spike to cool the 
tip and then rushes out laterally, as shown in Fig.1 (a). For an optimization design of the NaTPS, the spike-blunt body structure is able to avoid the shock/shock interaction occurs at the shoulder of the blunt body. The lateral jet becomes effective only when the attack angle is so large that the shock interaction point could approach to the blunt body surface.

The test model simplified from the NaTPS concept is schematically shown Fig.1 (b). The mode has a cylindrical body of diameter $D=80 \mathrm{~mm}$, with a hemispherical nose, and is measured $240 \mathrm{~mm}$ in length. A spike of $L=80 \mathrm{~mm}$ in length is installed at the stagnation point, which has a cylindrical body and a hemispherical nose. The spike is of a hollow structure with the outer diameter of $d=12 \mathrm{~mm}$ and inside diameter of $6 \mathrm{~mm}$. A half-circular orifice with a width of $1 \mathrm{~mm}$ is open on the spike body and located very close to the tip of the spike to produce lateral jets. The blunt body and the spike all are made of alloy steel 30CrMnSiA. Two rows of pressure transducers are located along the top and bottom generatrix of the model and each row has 15 orifices, respectively. The orifices are $0.1 \mathrm{~mm}$ in diameter.

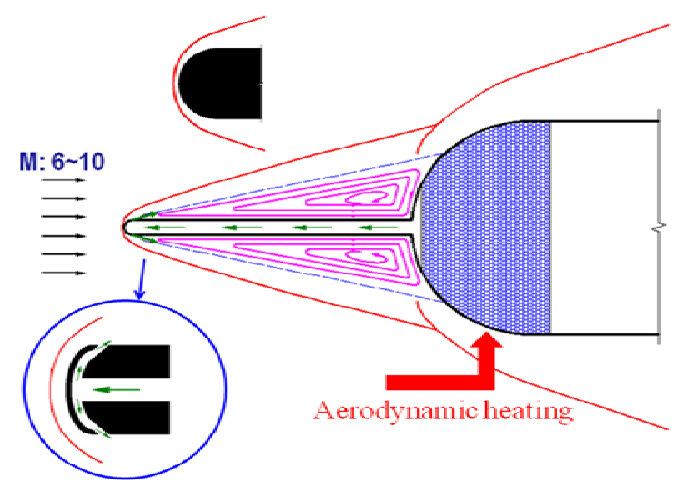

a) New concept of the NaTPS

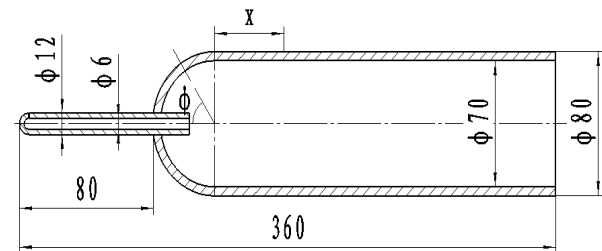

b) Test model for demostrating NaTPS

Figure 1. Schematic of the new concept and test model for the NaTPS.

The pressure measurement was conducted in the hypersonic wind tunnel of FD-07 in China Academy of Aerospace and Aerodynamics. The tunnel nozzle has a Ma=5.9332 and an exit diameter of $\Phi 500 \mathrm{~mm}$. Model installation is shown in Fig.2 and a series of runs were completed in the present study to investigate parameter effects of the jet total pressure $P^{*}$ and flight attack angles. Pressure measurements were carried out by using 8400 electronic pressure scanners and the flow around the test model were visualized by the schlieren system to demonstrate the shock wave structure around the NaTPS.

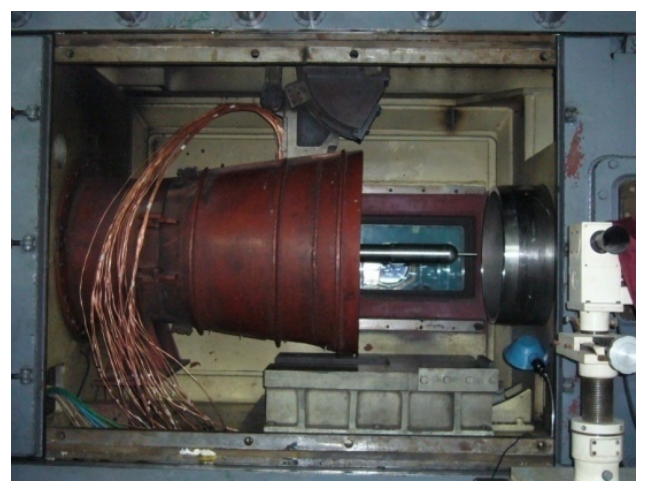

Figure 2. The test model installed in the hypersonic wind tunnel in CAAA, Beijing

3

American Institute of Aeronautics and Astronautics 
Numerical simulations were also conducted to be compared with the experimental data to investigate into the mechanism underlying shock wave reduction by lateral jets. The governing equations are the axisymmetric and three-dimensional compressible Navier-Stokes equations. The Spalart-Allmaras one-equation turbulence model is applied to simulate the turbulence mixing in the boundary layer and recirculation regions. The governing equations are solved with the two-order DCD scheme of the finite difference method ${ }^{26}$, and viscous terms are calculated with the two-order central differencing. Steger-Warming flux splitting method is employed to account for upwind effect ${ }^{27}$. The temporal integration is performed using the three-order TVD Runge-Kutta method ${ }^{28}$. The computational model is the same as the test one. The flow conditions are also taken to be the same as experiments. The wall boundary is assumed to be impermeable and non-slip conditions are applied along the isothermal surface of $\mathrm{T}_{\mathrm{w}}=500 \mathrm{~K}$. Due to the nature of the supersonic flow, the outflow boundary is linearly extrapolated from the interior of the computational domain and the inflow quantities are taken to be free stream values of flight at $30 \mathrm{~km}$ altitude. The conditions of lateral jet are assumed to be choked flow.

\section{Results and discussion}

\section{A. Role of the lateral jets}

The first test case is to demonstrate the role of lateral jets. Two experiments are carried out at zero attack angle, one with jet injection and the other without it. The schlieren photos are given in Fig.3. Figures 3 (a) shows the shock wave structure without lateral jet. It is observable that a conical shock wave is formed at the tip of the spike, accompanied with a boundary layer separation downstream caused by a positive pressure gradient along the spike. The angle of the conical shock wave is about 17 degree and a curved reattachment shock wave develops at the shoulder of the blunt body. The interaction point of the conical shock wave and the reattachment shock wave appears to be not at the body surface but very close to it. The schlieren photo with lateral jet injection is given in Fig.3 (b). It is evident that the lateral jet has a strong influence on the flowfield structure. The conical shock wave angle is first increased up to 60 degree by the lateral jet, and then decreases finally to about 30 degree. The conical shock wave in the upper half flowfield is pushed away from the blunt body so that shock interaction point could move away from the blunt body surface. The lateral jet in conjunction with the aero-spike prevents occurring of the shock/shock interaction on the shoulder of the blunt body.

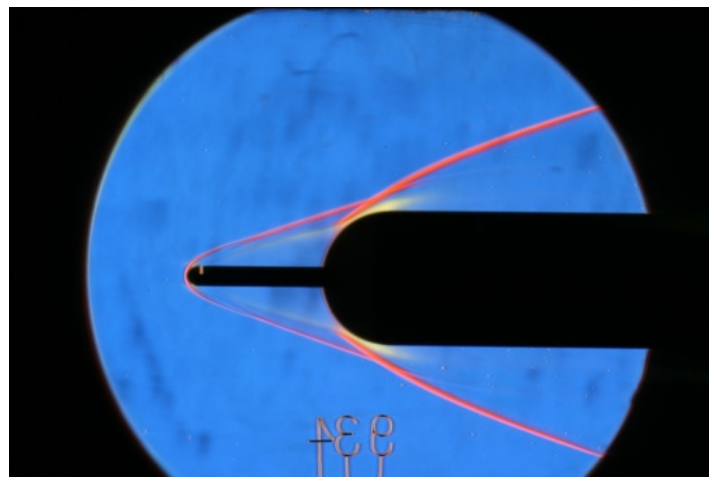

a) Experimental photo without lateral jets

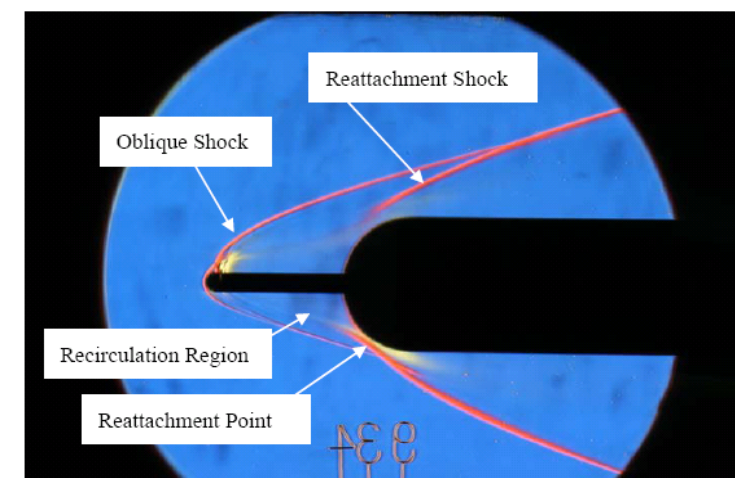

b) Experimental photo with lateral jets

Figure 3. Flow visualization for the test model without and with lateral jets at zero attack angle for Mach number 6

\section{B. Effects of the lateral jets on flowfiled at non-zero attack angle}

The second test case is to investigate the effects of the lateral jets on the flowfiled structure at an attack angle. Two experiments are carried out at $4^{\circ}$ attack angle, one with jet injection and the other without it. The schlieren photos are given in Fig.4. Figure 4 (a) shows the test case at $4^{\circ}$ attack angle, where the conical shock wave impinges on the shoulder of the blunt body and interacts with the reattachment shock wave on the windward side. This image shows that the spike-blunt body structure cannot avoid shock/shock interaction that produces very high peak pressure and heat flux at the reattachment point. The heat flux has been demonstrated to be ten times higher than that at the stagnation point for decades. This result reveals the key phenomenon from which it is understood that why the aerodynamic performance of aero-spike becomes worse at non-zero attack angle. Setting up the test model at $-4^{\circ}$ attack angle, the experiment is repeated when a lateral jet is applied on the windward side. The flow visualization result is shown in Fig.4 (b). It is clearly seen that the lateral jet increases the angle of the conical shock wave and acts to push it away from the blunt body. The reattachment shock wave also becomes weaker and the shock/shock 
interaction point is observed to be far away from the blunt body surface. As a result, both the peak pressure and heat flux decreases greatly at the reattachment region. This result demonstrates well that the lateral jet works perfectly at non-zero attack angles and maintains the good performance of the spike-blunt body structure for wave drag and heat flux reduction.

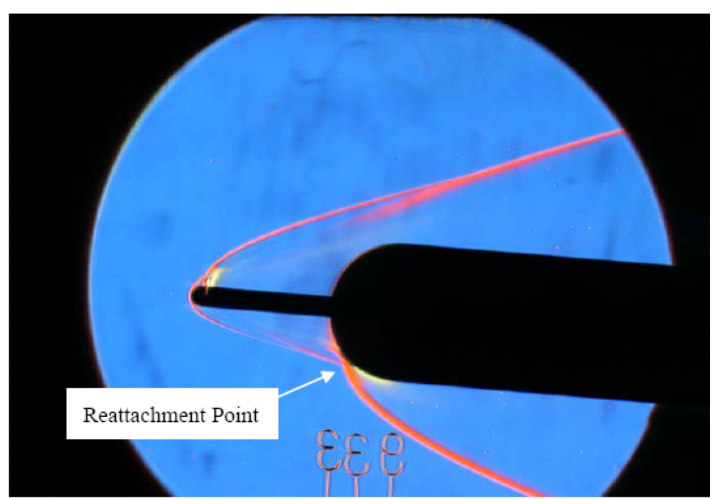

a) Photo without lateral jets at $4^{0}$ attack angle

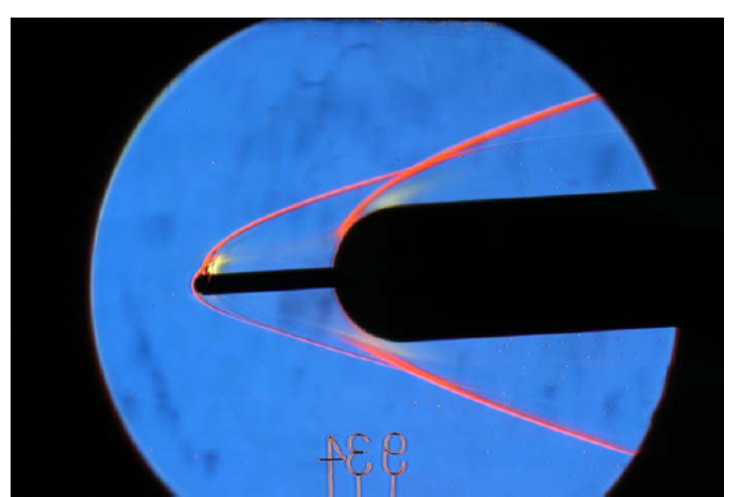

b) Photo with lateral jets at $-4^{0}$ attack angle

Figure 4. Flow visualization for the test model without and with lateral jets at $4^{\circ}$ attack angle for Mach number 6

The pressure measurements were carried out when doing the above mentioned two test cases. Figure 5 depicts the comparison of the pressure distributions along the generatrix of the test model with and without lateral jets at $4^{\circ}$ and $-4^{\circ}$ degree attack angles, respectively. The $\mathrm{x}$-coordinate is the ratio of the arc length on the blunt body surface measured from the geometric stagnation point to the blunt body diameter. It is evident that for the blunt body with and without lateral jets, the peak pressure occurs at the reattachment region because of the effect of the shock/shock interaction and decreases rapidly along the surface of the blunt body. Figure 5 (a) shows that, at a zero attack angle, the peak pressure without lateral jet is about $26 \mathrm{kPa}$, while the peak pressure with lateral jet is about $9 \mathrm{kPa}$. The peak pressure is reduced by $65 \%$ by the lateral jet injection. As a result, the shock wave drag reduction inferred from the pressure measurements is $33 \%$. The experimental result at $4^{\circ}$ attack angle is shown in Fig.5 (b). The peak pressure without lateral jet is $90 \mathrm{kPa}$ and is much higher than the stagnation pressure of $59.8 \mathrm{kPa}$. This is due to the effect of shock/shock interaction. The peak pressure with lateral jet injection is about $26 \mathrm{kPa}$ and reduced by $72 \%$. This peak pressure is higher than that shown in Fig.5 (a), but still lower than the stagnation pressure. The comparison of pressure distributions quantitatively demonstrated that the lateral jet injection is very effective in modifying the shock wave structure, mitigating the shock/shock interaction, and reducing the shock wave drag and heat flux.

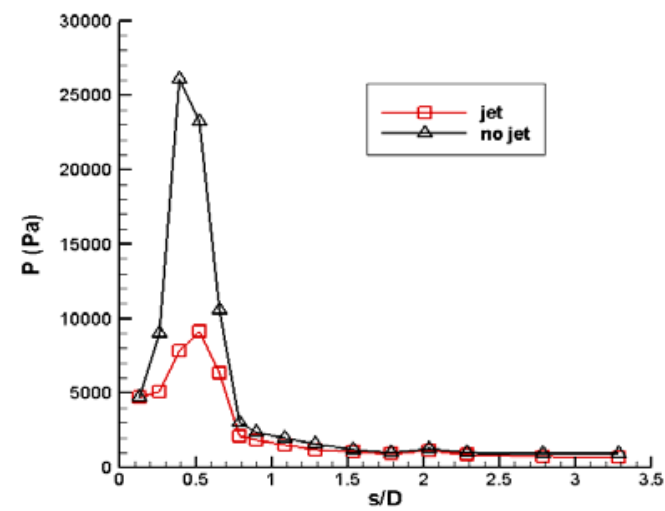

a) Pressure profiles at zero attack angle

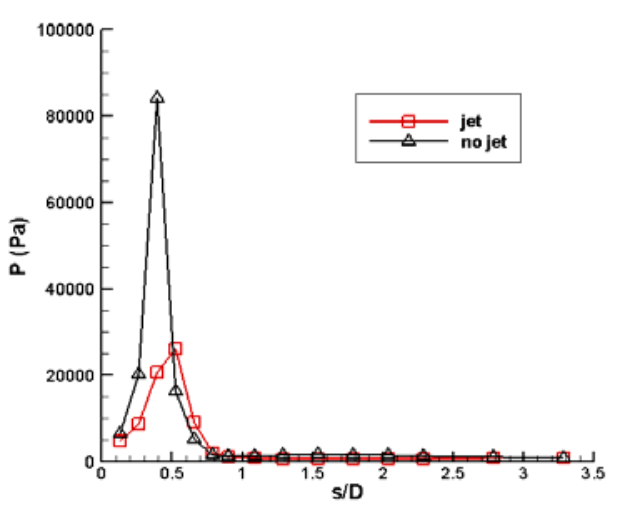

b) Pressure profiles at 4 degree angle of attack

Figure 5. Comparisons of pressure profiles along the generatrix of the test model with and without lateral jets

\section{Numerical and experimental result validation}

Axisymmetric numerical simulations were conducted to be compared with the experiments for result validation. Some relative results are shown in Fig.6. The shock structure shown in Figure 6 (a) seems to be identical to that in the experiment photo shown in Fig.3 (a), such as the conical shock angel and the reattachment shock wave. The comparisons of the experimental pressure distribution with numerical simulations are given in Fig.6 (b) and very 
good agreement is also achieved. The validation does not only verify the numerical results, but also demonstrate that the experimental data are reliable.

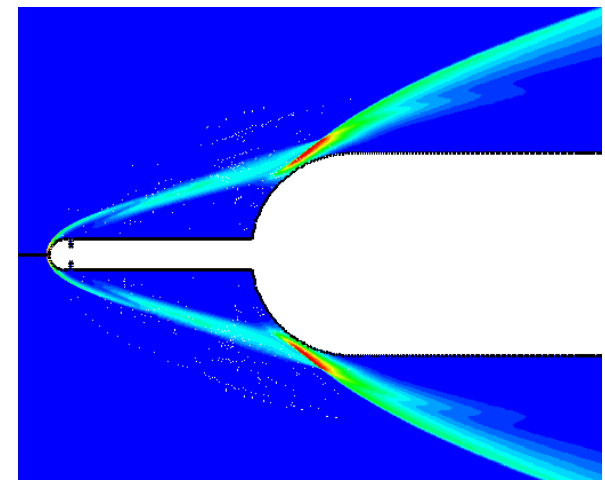

a) Pressure distribution at zero attack angle

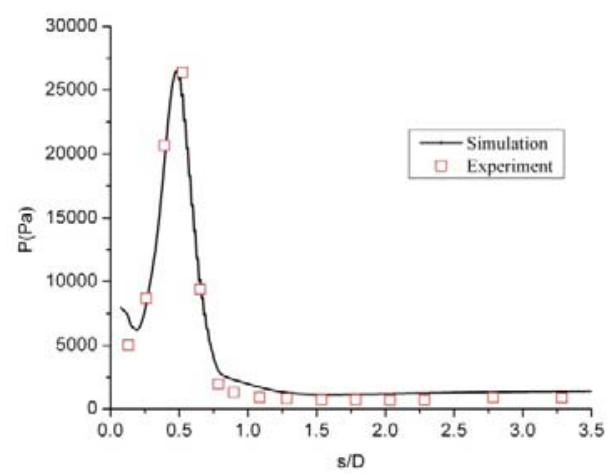

b) Numerical and experimental pressure profiles

Figure 6. Numerical simulations and the comparison of experimental pressure profiles with numerical results at zero attack angle without lateral jet

\section{Role of the recirculation region}

Three-dimensional numerical simulations were conducted to investigate into the mechanism underlying the NaTPS. The results of the case at the $4^{\circ}$ angle attack both with and without lateral jets are presented in Figs. 7 and 8 , respectively. It can be seen from density distributions in Fig.7 (a) that for the case without lateral jet, the conical shock wave on the windward side impinges upon the blunt body and interacts with the reattachment shock wave, resulting in a high pressure and temperature region around the interaction point. It is because that a part of the gas flow on the windward side moves to the leeward side and the corresponding recirculation region shrinks, as shown in Fig.8 (a). The spike-blunt body structure cannot maintain a recirculation region on the windward side, which should be bigger enough to avoid shock interaction at $-4^{\circ}$ angle attack.

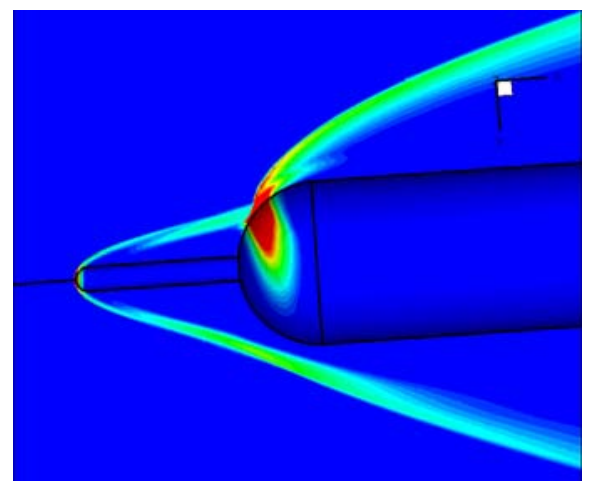

a) Density distribution without lateral jet

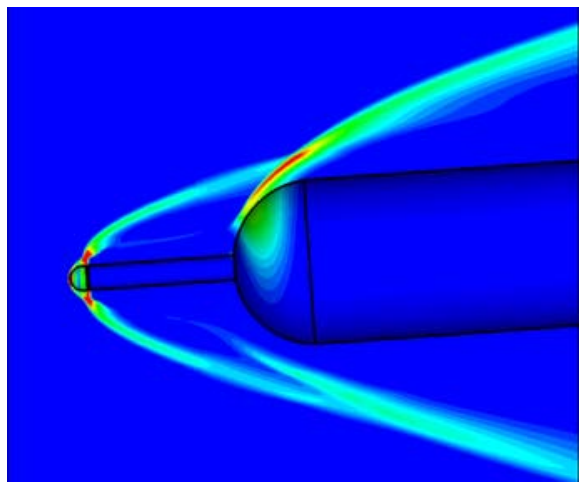

b) density distribution with lateral jets

Figure 7. Contours of density at $-4^{0}$ attack angle for test cases with and without lateral jet injections

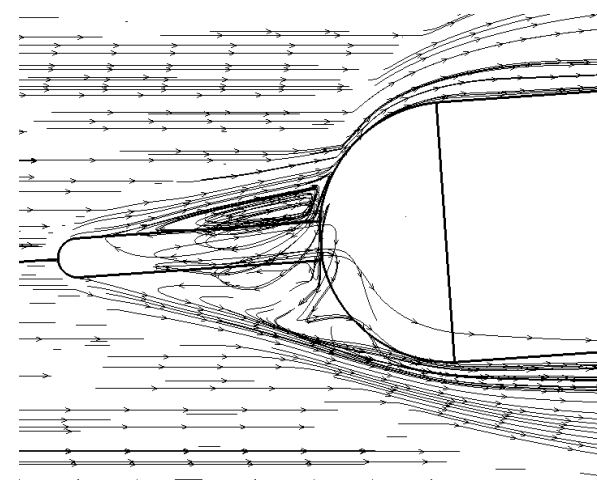

a) Streamlines without lateral jet

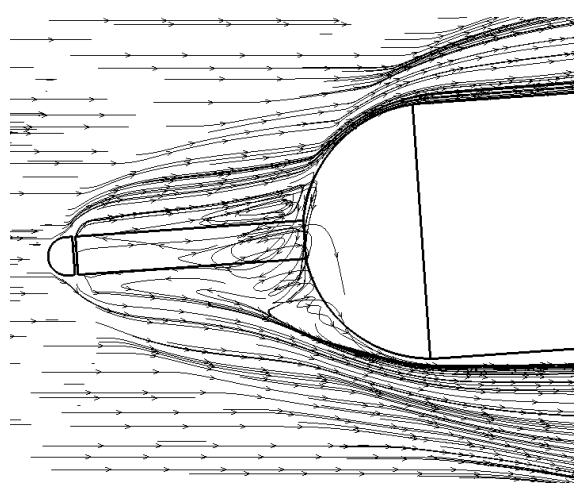

b) Streamlines with lateral jets

Figure 8. Streamlines at $-4^{\circ}$ attack angle for test cases with and without lateral jet injections

6

American Institute of Aeronautics and Astronautics 
In contrast, for the case with lateral jets as shown in Fig.7 (b), the lateral jet deflects the flow behind the conical shock wave effectively and pushes it away from the blunt body. The interaction of the conical shock wave with the reattachment shock wave does not occur on the surface of the blunt body. Figure 8 (b) shows that the conical shock wave angle is increased and the leak of the gas flow from the windward side to the leeward side is weakened. In this case, the peak pressure and heat flux at the reattachment region are decreased significantly and accordingly both shock wave drag and aerodynamic heat flux are also reduced significantly.

\section{Conclusion}

In this study, a new concept of shock wave drag reduction and thermal protection for hypersonic vehicles was proposed, which was named as the Non-ablative Thermal Protection System (NaTPS). In the NaTPS, an aero-spike recasts the bow shock in front of a blunt body into a conical shock wave. A lateral jet injecting at the tip of the spike works to increase the conical shock wave angle and pushes it away from the blunt body when a pitching angle exists during flight to mitigate the shock/shock interaction on the shoulder of the blunt body. As a result, the shock wave drag and the thermodynamic payloads on hypersonic vehicles are significantly reduced by the NaTPS. The flow visualization, pressure measurements and numerical simulations were conducted to verify this new concept. The experimental schlieren photos show that the conical shock wave generated at the tip of the spike is pushed away from the blunt body by the lateral jet and the shock/shock interaction on the shoulder is eliminated at the $4^{\circ}$ attack angle. The peak pressure at the reattachment region is reduced by $65 \%$ and the shock wave drag inferred from the pressure measurements is reduced by $33 \%$. The numerical results were demonstrated to be in good agreement with experimental data. The further three-dimensional simulation reveals that the lateral jet effectively deflects the flow downstream, modifies the shock structure of the flowfield, and keeps the recirculation region in a proper size to avoid the shock interaction. The excellent performance of the NaTPS for shock wave drag reduction and thermal protection for hypersonic vehicles is well demonstrated and the new concept seems to be of potential importance for engineering applications.

\section{Acknowledgments}

The authors would like to acknowledge the National Natural Science Foundation of China who provides funding for this research under the project number of 90916028.

\section{References}

${ }^{1}$ Anderson, J. D., Hypersonic and High Temperature Gas Dynamics, McGraw-Hill, New York, 1989.

${ }^{2}$ Bushnell, D. M., "Shock Wave Drag Reduction," Annu. Rev. Fluid. Mech., Vol. 36, 2004, pp. 81-96.

${ }^{3}$ Crawford, D. H., "Investigation of the Flow Over a Spiked-Nose Hemisphere-Cylinder at a Mach Number of 6.8," NASA TN-D118, 1959.

${ }^{4}$ Hutt, C. R., and Howe, A. J., "Forward Facing Spike Effects of Bodies of Different Cross Section in Supersonic Flow," The Aeronautical Journal of the Royal Aeronautical Society, Vol. 93, No. 6, 1989, pp. 229-234.

${ }^{5}$ Milićev, S. S., Pavlović, M. D., Ristić, S., and Vitić, A., "On the Influence of Spike Shape at Supersonic Flow Past Blunt Bodies," Facta Universitatis, Series: Mechanics, Automatic Control and Robotics, Vol. 3, No. 12, 2002, pp. 371-382.

${ }^{6}$ Menezes, V., Saravanan, S., Jagadeesh, G. and Reddy, K. P. J., "Experimental Investigations of Hypersonic Flow Over Highly Blunted Cones with Aerospikes," AIAA Journal, Vol. 41, No. 10, 2003, pp. 1955-1961.

${ }^{7}$ Reding, J. P., Guenther, R. A., and Richter, B. J., "Unsteady Aerodynamic Consideration in the Design of a Drag-Reduction Spike," Journal of Spacecraft and Rocket, Vol. 14, No.1, 1977, pp. 54-60.

${ }^{8}$ Stadler, J. R., and Nielsen, H. V., "Heat Transfer from a Hemispherical Cylinder Equipped with Flow-Separation Spikes," NACA TN-3287, Sept. 1954.

${ }^{9}$ Chapman, D. R., "A Theoretical Analysis of Heat Transfer in Region of Separated Flow,” NACA TN-3792, June, 1956.

${ }^{10}$ Mehta, R. C., "Numerical Heat Transfer Study Over Spiked Blunt Bodies at Mach 6.8," J. Spacecraft, Vol. 37, No. 5, 2000, pp.700-703.

${ }^{11}$ Remeo, D. J., and Sterrett, J. R., "Exploratory Investigation of the Effect of a Forward-Facing Jet on the Bow Shock of a Blunt Body in a Mach Number 6 Free Stream," NASA TN D-1605, 1963.

${ }^{12}$ Finley, P. J., "The Flow of a Jet From a Body Opposing a Supersonic Freestream,” J. Fluid Mech., Vol. 26, 1966, pp. 337368.

${ }^{13}$ Meyer, B., Nelson, H. F., and Riggins, D. W., "Hypersonic Drag and Heat-Transfer Reduction Using a Forward-Facing Jet," Journal of Aircraft, Vol. 38, No. 4, 2001, pp. 680-686.

${ }^{14}$ Venukumar, B., Jagadeesh G., and Reddy, K. P. J., "Counterflow Drag Reduction by Supersonic Jet for a Blunt Body in Hypersonic Flow," Physics of Fluids, Vol. 18, 118104, 2006.

${ }^{15}$ Sahoo, N., "Film Cooling Effectiveness on a Large Angle Blunt Cone Flying at Hypersonic Speed," Physics of Fluids, Vol. 17, 036102, 2005. 
${ }^{16}$ Riggins, D., Nelson, H. F., and Johnson, E., "Blunt-Body Wave Drag Reduction Using Focused Energy Deposition,” AIAA Journal,Vol. 37, No. 4, April 1999.

${ }^{17}$ Kremeyer, K., Sebastian, K., and Shu, C.-W., "Computational Study of Shock Mitigation and Drag Reduction by Pulsed Energy Lines,” AIAA Journal, Vol. 44, No. 8, August 2006.

${ }^{18}$ Knight, D., "Survey of Aerodynamic Drag Reduction at High Speed by Energy Deposition," Journal of Propulsion and Power, Vol. 24, No. 6, November-December, 2008.

${ }^{19}$ Bivolaru, D., and Kuo, S. P., "Aerodynamic Modification of Supersonic Flow Around Truncated Cone Using Pulsed Electrical Discharges,” AIAA Journal , Vol. 43, No. 7, July 2005.

${ }^{20}$ Kuo, S. P., "Plasma Mitigation of Shock Wave: Experiments and Theory," Shock Waves, Vol. 17, 2007, pp. 225-239.

${ }^{21}$ Golovitch V.I., and Hansson J., "Some Trends in Improving Hypersonic Vehicles Aerodynamic and Propulsion," AIAA IS090, 1998.

${ }^{22}$ Yuriev, A. S., Pirogov, S. Y., Savischenko, N. P., Leonov, S. B., and Ryizhov, E. V., "Numerical and Experimental Investigation of Pulse Repetitive Energy Release Upstream Body under Supersonic Flow," AIAA Paper 2002-2730, 2002.

${ }^{23}$ Zaidi, S. H., Shneider, M. N., Mansfield, D. K., Ionikh, Y. Z., and Miles, R. B., "Influence of Upstream Pulsed Energy Deposition on a Shock Wave Structure in Supersonic Flow," AIAA Paper 2002-2703,2002.

${ }^{24}$ Jiang, Z. L., Liu, Y. F., Han, G. L., and Zhao, W., "Experimental Demonstration of a New Concept of Drag Reduction and Thermal Protection for Hypersonic Vehicles," Acta Mech Sin, Vol. 25, No. 3, pp. 417-419.

${ }^{25}$ Anderson, J. D., Modern Compressible Flow, McGraw-Hill, New York, 1990.

${ }^{26}$ Jiang, Z. L., “On Dispersion-Controlled Principles For Non-Oscillatory Shock-Capturing Schemes,” Acta Mechanica Sinica, Vol. 20, No. 1, 2004, pp.1-15.

${ }^{27}$ Steger, J. L., and Warming, R. F., "Flux Vector Splitting of the Inviscid Gasdynamic Equations with Application to FiniteDifference Methods," J. Comput. Phys., Vol. 40, 1981, pp. 263-293.

${ }^{28}$ Shu, C.-W., and Osher, S., "Efficient Implementation of Essentially Nonoscillatory Shock-Capturing Schemes II," J. Comput. Phys., Vol. 83, 1989, pp. 32-78. 